\title{
Physical inactivity is associated with narrower lumbar intervertebral discs, high fat content of paraspinal muscles and low back pain and disability
}

Andrew J Teichtahl ${ }^{1,2}$, Donna M Urquhart ${ }^{1}$, Yuanyuan Wang ${ }^{1}$, Anita E Wluka', Richard O'Sullivan ${ }^{3,4}$, Graeme Jones $^{5}$ and Flavia M Cicuttini $i^{*}$

\begin{abstract}
Introduction: Although physical inactivity has been associated with numerous chronic musculoskeletal complaints, few studies have examined its associations with spinal structures. Moreover, previously reported associations between physical activity and low back pain are conflicting. This study examined the associations between physical inactivity and intervertebral disc height, paraspinal fat content and low back pain and disability.

Methods: Seventy-two community-based volunteers not selected for low back pain underwent magnetic resonance imaging (MRI) of their lumbosacral spine (L1 to S1) between 2011 and 2012. Physical activity was assessed between 2005 and 2008 by questionnaire, while low back pain and disability were assessed by the Chronic Pain Grade Scale at the time of MRI. Intervertebral disc height and cross-sectional area and fat content of multifidus and erector spinae were assessed from MRI.
\end{abstract}

Results: Lower physical activity levels were associated with a more narrow average intervertebral disc height $(\beta-0.63$ $\mathrm{mm}, 95 \%$ confidence interval $(\mathrm{Cl})-1.17 \mathrm{~mm}$ to $-0.08 \mathrm{~mm}, P=0.026)$ after adjusting for age, gender and body mass index (BMI). There were no significant associations between physical activity levels and the cross-sectional area of multifidus or erector spinae. Lower levels of physical activity were associated with an increased risk of high fat content in multifidus (odds ratio $(\mathrm{OR}) 2.7,95 \% \mathrm{Cl} 1.1$ to $6.7, P=0.04)$ and high-intensity pain/disability $(\mathrm{OR}=5.0$, $95 \% \mathrm{Cl} 1.5$ to $16.4, P=0.008$ ) after adjustment for age, gender and BMI.

Conclusions: Physical inactivity is associated with narrower intervertebral discs, high fat content of the multifidus and high-intensity low back pain and disability in a dose-dependent manner among community-based adults. Longitudinal studies will help to determine the cause and effect nature of these associations.

\section{Introduction}

The Global Burden of Diseases study ranked low back pain the leading cause of disability of 291 conditions examined, and sixth in terms of disability-adjusted life years increasing from 58.2 million in 1990 to 83 million in 2010 [1]. Targeting modifiable risk factors associated with low back pain may therefore be helpful in reducing the burden of this disease.

\footnotetext{
* Correspondence: flavia.cicuttini@monash.edu

'Department of Epidemiology and Preventive Medicine, School of Public Health and Preventive Medicine, Monash University, Alfred Hospital, 99 Commercial Road, Melbourne, VIC 3004, Australia

Full list of author information is available at the end of the article
}

Although physical inactivity has been associated with numerous chronic musculoskeletal complaints [2], two systematic reviews have shown the evidence linking physical activity and low back pain to be conflicting $[3,4]$. While one systematic review of 15 studies [3] found only one study demonstrating a significant positive relationship between physical inactivity and low back pain [5], the populations investigated were diverse, comprising both school children and adults. A more recent systematic review of 17 studies concluded that the relationship between physical activity and low back pain was too heterogeneous to reach any conclusion [4].

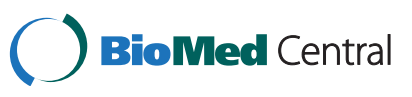

(C) 2015 Teichtahl et al.; licensee BioMed Central. This is an Open Access article distributed under the terms of the Creative Commons Attribution License (http://creativecommons.org/licenses/by/4.0), which permits unrestricted use, distribution, and reproduction in any medium, provided the original work is properly cited. The Creative Commons Public Domain Dedication waiver (http://creativecommons.org/publicdomain/zero/1.0/) applies to the data made available in this article, unless otherwise stated. 
While studies have investigated, albeit inconclusively, the association between physical activity and back pain $[3,4]$, the associations between physical activity and spinal structures have not been widely studied. Several studies have shown that mechanical stimulation is required to maintain intervertebral disc integrity [6,7], and that forced immobility is associated with muscle atrophy, such as a marked reduction in muscle size observed after spinal cord injury [8]. It may therefore be that physical inactivity plays a role in the pathogenesis of these structural abnormalities. Which structures are of clinical significance is, however, speculative, although intervertebral disc height and paraspinal muscle size and fat content have been shown to be associated with low back pain [9-11].

The aim of this study was to examine the associations between physical activity levels among community-based adults and 1) lumbar spine structures including intervertebral disc height and paraspinal muscle properties (cross-sectional area (CSA) and fat content), as well as 2) low back pain and disability. We hypothesised that physical inactivity would be independently associated with a narrow intervertebral disc, less CSA and high fat content of paraspinal muscles, as well as high-intensity low back pain and disability.

\section{Methods}

\section{Participants}

Seventy-two community-based individuals recruited through local media and weight loss clinics were examined as part of a study of obesity and musculoskeletal health [12]. Participants were recruited without reference to whether they had or did not have low back pain. Participants were excluded if there was a history of any arthropathy diagnosed by a medical practitioner (including inflammatory arthropathies or mechanical joint derangements), previous significant injury requiring non-weight-bearing therapy or prescribed analgesia, malignancy, contraindication to magnetic resonance imaging (MRI) or inability to understand English. The study was approved by the Human Research Ethics Committees of the Alfred Hospital and Monash University. All participants gave written informed consent.

\section{Physical activity level assessment}

Physical activity levels for each participant were made available from a questionnaire conducted between 2005 and 2008 that has previously been used to assess exercise levels in healthy adults [13]. Participants were asked over the 14 days prior the questionnaire, how many days they had participated in strenuous activity for at least 20 minutes duration. Strenuous was defined as activity leading to sweating or shortness of breath and examples such as swimming, tennis, netball, athletics and running were listed. It has been shown that this intensity of exercise captured by questionnaire tends to better approximate habitual physical activity [14]. From these data, three categories of physical activity were extrapolated: active ( 9 to 14 days), moderately active (1 to 8 days) and inactive (0 days).

\section{Magnetic resonance imaging}

MRI was performed (2011 to 2012) using a 3.0T magnetic resonance unit (MAGNETOM Verio, A Tim System; Siemens, Erlangen, Germany). The participant was positioned in the supine position and the following scans were performed: (1) sagittal T1 images from T12 to the sacrum (repetition time: $670 \mathrm{~ms}$; echo time: 12 ms, slice thickness: $4 \mathrm{~mm}$ ), (2) sagittal T2 images from T12 to sacrum (repetition time: 3,000 to 3,600 ms; echo time: 87 to $114 \mathrm{~ms}$, slice thickness: $4 \mathrm{~mm}$ ), and (3) axial T2 images from L1 to S1 (repetition time: 3,000 to 3,600 ms; echo time: 87 to $114 \mathrm{~ms}$, slice thickness: $4 \mathrm{~mm}$ ).

\section{Intervertebral disc height}

Intervertebral disc height of the lumbar spine was measured on mid-sagittal MR images from the middle of the superior border of the disc to the middle of the inferior border of the disc with the inclusion of both end plates. One trained observer measured the disc height in duplicate, one week apart, blinded to the characteristics of the participants. The intra-rater reliability of the disc height measures at each vertebral level was high, with intra-class correlation coefficients (ICCs) ranging from 0.94 to 0.98 . The average disc height was calculated by summing L1/2, L2/3, L3/4, L4/5, and L5/S1 intervertebral disc height and dividing by five.

\section{Muscle cross-sectional area and fat content}

Paraspinal muscle CSA and fat content were determined at the level of the L3/L4 intervertebral disc. The CSA of multifidus and erector spinae were measured by outlining the border of each muscle using a tool in OsiriX. The measurements were repeated by the same observer, two weeks apart. To avoid bias, the observer was blinded to the first measurement when performing the second measurement. The intra-observer reliability (ICC) was 0.99 .

Hyperintense regions within the paraspinal muscles observed on T2 axial images were considered fatty tissue [15] and categorised based on a previously validated grading method; grade 0: no fat, grade 1: 1 to $10 \%$ fat, grade 2: 11 to $50 \%$ fat and grade $3:>50 \%$ fat [15]. The intra-observer reliability (ICC) for the paraspinal muscle fat content for both multifidus and erector spinae was 0.99. High fat content was defined as greater than $50 \%$ of the muscle (see Figure 1). 


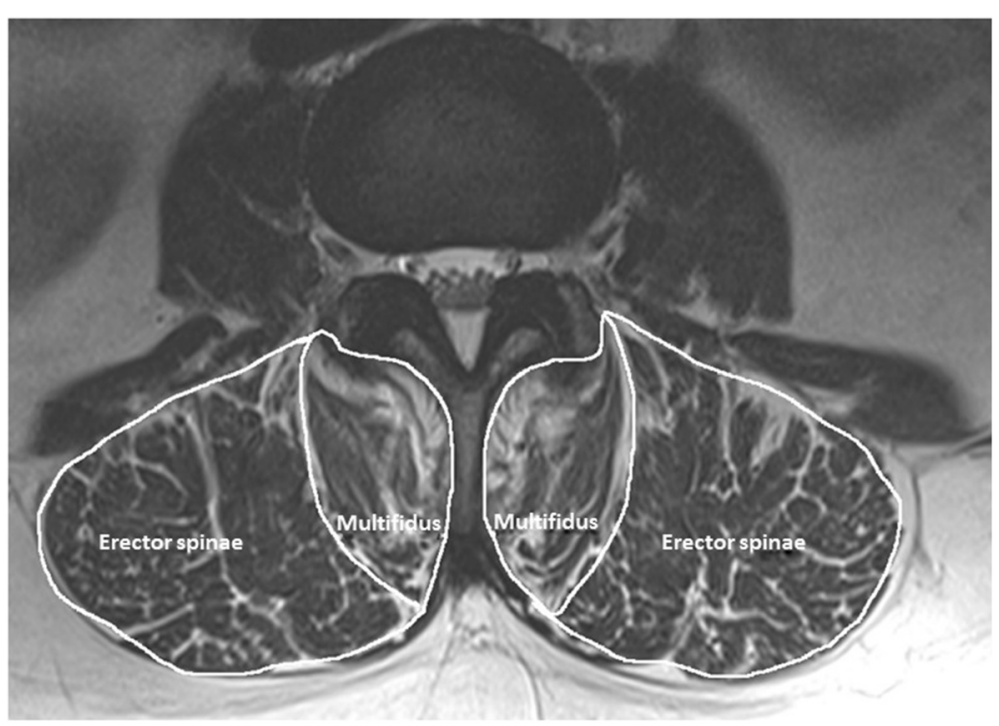

Figure 1 An example of high fat (>50\%) content of multifidus but not erector spinae.

\section{Anthropometric data}

Height was measured to the nearest $0.1 \mathrm{~cm}$ using a stadiometer. Weight was measured to the nearest $0.1 \mathrm{~kg}$ using a single pair of electronic scales. Body mass index (BMI) $\left(\mathrm{kg} \mathrm{m}^{-2}\right)$ was calculated.

\section{Chronic pain and disability}

The Chronic Pain Grade Questionnaire was administered at the time of MRI in 2011 to 2012 to obtain information on low back pain intensity over the past six months. The Chronic Pain Grade Questionnaire is a reliable and valid instrument for use in population surveys of low back pain [16,17]. The questionnaire includes seven questions from which a pain intensity and disability subscale score are calculated. Subscale scores for pain intensity and disability are combined to calculate a chronic pain grade that enables classification of chronic pain into five hierarchical categories: grades 0 (no pain) to 4 (high disability, severely limiting) as previously described [16,17]. High-intensity pain/disability was defined as being of either grade 2 (low disability but high intensity), grade 3 (high disability, moderately limiting) or grade 4 (high disability, severely limiting).

\section{Statistical analyses}

The inactive, moderately active and active groups were compared. The F test was used to determine the pairwise comparison among the estimated marginal means for continuous data, chi-squared tests for categorical data and the Fisher's exact test for chronic pain grade data. Multiple linear regression analyses were used to examine the associations between physical activity levels and intervertebral disc height, adjusted for age, gender and BMI. Multiple linear regression analyses were also employed to examine the relationship between physical activity levels and paraspinal muscle CSA, adjusted for age, gender, BMI and fat content. Binary logistic regression analyses were used to examine the relationships between physical activity levels and the risk of high $(>50 \%)$ paraspinal muscle fat content and high-intensity pain/ disability, with adjustment made for age, gender and BMI. We chose to examine muscle variables on the left side only for standardisation purposes and to avoid multiple testing. A P value of less than 0.05 (two-tailed) was regarded as statistically significant. All analyses were performed using the IBM SPSS statistical package (standard version 20.0 IBM Corp., Armonk, NY, USA).

\section{Results}

The majority of participants in the study were female (68.1\%), and the average BMI was in the overweight category $\left(29.2 \mathrm{kgm}^{-2}\right)$. Approximately $21 \%$ of the participants were inactive, and $53 \%$ and $26 \%$ had moderate and high levels of physical activity respectively.

Participant characteristics $(\mathrm{N}=72)$ are shown in Table 1, according to their activity levels. People with higher activity levels had a smaller weight $(P=0.02)$ and BMI $(P=0.002)$ than their less physically active counterparts. The average intervertebral disc height in the lumbosacral (L1 to S1) spine was narrower the more physically inactive a person was (inactive: 10.2 (standard error of the mean (SEM) 4.1) mm, moderately active 10.6 (SEM 2.6) $\mathrm{mm}$ and active 11.8 (SEM 3.6) $\mathrm{mm}, P=0.01$ for difference among the three groups). High fat content of multifidus was more prevalent in the less physically active groups (inactive: 60\%, 
Table 1 Participant characteristics

\begin{tabular}{|c|c|c|c|c|}
\hline & $\begin{array}{l}\text { Inactive } \\
\text { (0 days) } \\
\mathrm{N}=15\end{array}$ & $\begin{array}{l}\text { Moderately active } \\
\text { (1 to } 8 \text { days) } \\
\mathrm{N}=38\end{array}$ & $\begin{array}{l}\text { Active } \\
\text { (9 to } 14 \text { days) } \\
\mathrm{N}=19\end{array}$ & $P$ \\
\hline Age (years) & $47.2(1.3)$ & $45.1(0.7)$ & $46.5(1.5)$ & 0.29 \\
\hline Gender ( $n, \%$ female) ${ }^{* *}$ & $13(86.7)$ & $26(68.4)$ & $10(52.6)$ & 0.11 \\
\hline Weight $(\mathbf{k g})^{*}$ & $99.0(3.6)$ & $96.5(2.1)$ & $84.4(4.3)$ & 0.02 \\
\hline Height $(\mathrm{m})^{*}$ & $1.67(0.01)$ & $1.67(0.01)$ & $1.70(0.02)$ & 0.21 \\
\hline BMI $\left(\mathrm{kgm}^{-2}\right)^{*}$ & $35.7(1.3)$ & $34.8(0.8)$ & $29.3(1.5)$ & 0.002 \\
\hline \multicolumn{5}{|c|}{ Intervertebral disc height (L1 to S1) $(\mathrm{mm})^{*}$} \\
\hline Average & $10.2(4.1)$ & $10.6(2.6)$ & $11.8(3.6)$ & 0.01 \\
\hline \multicolumn{5}{|l|}{ Paraspinal muscle properties } \\
\hline \multicolumn{5}{|l|}{$\operatorname{CSA}\left(\mathrm{cm}^{2}\right)^{*}$} \\
\hline Multifidus & $6.9(0.4)$ & $6.9(0.3)$ & $7.2(0.4)$ & 0.76 \\
\hline Erector spinae & $19.7(1.1)$ & $20.5(0.7)$ & $21.7(1.0)$ & 0.41 \\
\hline \multicolumn{5}{|l|}{ High fat content, $\mathrm{n}(\%)^{* *}$} \\
\hline Multifidus & $9(60.0)$ & $13(34.2)$ & $3(15.8)$ & 0.03 \\
\hline Erector spinae & $5(33.3)$ & $7(18.4)$ & $1(5.3)$ & 0.11 \\
\hline \multicolumn{5}{|c|}{ Chronic low back pain grade, $\mathrm{n}(\%)^{* * *}$} \\
\hline Pain free & $3(20.0)$ & $6(15.8)$ & $5(26.3)$ & 0.049 \\
\hline Low disability, low intensity & $5(33.3)$ & $24(63.2)$ & $14(73.7)$ & \\
\hline Low disability, high intensity & $4(26.7)$ & $1(2.6)$ & $0(0.0)$ & \\
\hline High disability, moderately limiting & $1(6.7)$ & $4(10.5)$ & $0(0.0)$ & \\
\hline High disability, severely limiting & $2(13.3)$ & $3(7.9)$ & $0(0.0)$ & \\
\hline High pain/disability, $\mathrm{n}(\%)^{* * *}$ & $7(46.7)$ & $8(21.1)$ & $0(0.0)$ & 0.004 \\
\hline
\end{tabular}

moderately active: $34.2 \%$, active: $15.8 \% ; P=0.03)$. The chronic pain grade was greater in the less physically active groups $(P=0.049)$.

Lower physical activity level was associated with an increased risk of high-intensity pain/disability (odds ratio $(\mathrm{OR})=5.0,95 \%$ confidence interval $(\mathrm{CI}) 1.5$ to 16.4, $P=0.008$ ) after adjustment for age, gender and BMI (data not shown). Moreover, in univariate analyses, lower physical activity levels were associated with more narrow average lumbosacral intervertebral disc height $(\beta-0.78 \mathrm{~mm}, 95 \% \mathrm{CI}-1.31 \mathrm{~mm}$ to $-0.23 \mathrm{~mm}, P=$ 0.006). This association persisted after adjusting for age, gender and BMI ( $\beta-0.63 \mathrm{~mm}, 95 \% \mathrm{CI}-1.17 \mathrm{~mm}$ to -0.08 mm, $P=0.026)$.

The associations between activity levels and paraspinal muscle properties are shown in Table 2. There were no significant associations between physical activity levels and the CSA of multifidus or erector spinae. In univariate analyses, lower levels of physical activity were associated with an increased risk for high fat content in multifidus (OR 2.8, 95\% CI 1.3 to $6.3, P=0.01$ ). This relationship persisted after adjusting for age, gender and
BMI (OR 2.7, 95\% CI 1.1 to $6.7, P=0.04$ ). In univariate analyses, lower levels of physical activity were associated with an increased risk for high fat content in erector spinae (OR 2.7, 95\% CI 1.0 to $7.1, P=0.04$ ). This relationship failed to maintain statistical significance after

Table 2 The associations between lower levels of physical activity and paraspinal muscle properties

\begin{tabular}{|c|c|c|c|c|}
\hline \multicolumn{5}{|c|}{ Risk of high fat content } \\
\hline & Univariate & $P$ & Multivariate & $P$ \\
\hline & OR $(95 \% \mathrm{Cl})$ & & OR $(95 \% \mathrm{Cl})^{1}$ & \\
\hline Multifidus & $2.8(1.3,6.3)$ & 0.01 & $2.7(1.1,6.7)$ & 0.04 \\
\hline Erector spinae & $2.7(1.0,7.1)$ & 0.04 & $2.2(0.7,6.9)$ & 0.16 \\
\hline \multicolumn{5}{|c|}{ Cross-sectional area $\left(\mathrm{cm}^{2}\right)$} \\
\hline & Univariate & $P$ & Multivariate & $P$ \\
\hline & $\beta(95 \% \mathrm{Cl})$ & & $\beta(95 \% \mathrm{Cl})^{2}$ & \\
\hline Multifidus & $0.15(-0.43,0.73)$ & 0.61 & $0.09(-0.48,0.66)$ & 0.76 \\
\hline Erector spinae & $-1.01(-2.53,0.50)$ & 0.19 & $-0.58(-2.07,0.91)$ & 0.44 \\
\hline
\end{tabular}

${ }^{1}$ Risk of fat infiltration adjusted for age, gender, and $\mathrm{BMI} ;{ }^{2}$ cross-sectional area adjusted for age, gender, $\mathrm{BMI}$ and fat content. $\mathrm{OR}$, odds ratio; $\mathrm{Cl}$, confidence interval; BMI, body mass index. 
adjusting for age, gender and BMI (OR 2.2, 95\% CI 0.7 to $6.9, P=0.16$ ). When the low back pain grade was added to the multivariate analyses, results were unchanged (data not shown).

\section{Discussion}

This study has demonstrated a dose-response relationship between physical inactivity and structural abnormalities in the lumbosacral spine, including narrow intervertebral disc height and an increased risk for high $(>50 \%)$ multifidus fat content. Moreover, there was a dose-response relationship between physical inactivity and the risk for high-intensity low back pain/disability. These data provide the first evidence that among community-based adults, physical inactivity is associated with deleterious changes in the structure of the lumbosacral spine.

Intervertebral disc narrowing is a feature of degenerative disc disease, suggested by some to be the single most important structural risk factor for low back pain [18]. Nevertheless, degenerative disc disease is an umbrella term and classification systems vary across studies [19]. Since lumbar intervertebral disc height has been associated with low back pain [11], it is likely to represent a clinically important structural endpoint that is not reliant on a whole host of other radiological features, such as signal change in the intervertebral disc. In this study, we have demonstrated a dose-response association between physical activity and the average intervertebral disc height in the lumbosacral spine. Moreover, in this study, disc height has been assessed quantitatively and treated as a continuous variable, providing a more sensitive assessment than previous methods that have employed qualitative descriptors such as 'normal to slightly decreased' intervertebral disc height as a measure of disc degeneration [20]. Why low levels of physical activity may be associated with a reduction in intervertebral disc height is speculative. One possibility is that low levels of physical activity may result in inadequate mechanical stimulation, a factor important in maintaining intervertebral disc integrity [6,7]. It is also possible that a narrower intervertebral disc may cause pain and resultant low levels of physical activity. However, when we adjusted for the low back pain grade, results were unchanged (data not shown), suggesting that low levels of physical activity are associated with more narrow intervertebral disc height, independent of pain and disability.

Forced immobility has previously been shown to be associated with muscle atrophy, such as a marked reduction in muscle size observed after spinal cord injury [8]. Such data lead us to hypothesise that physical inactivity would be associated with less paraspinal muscle CSA. Nevertheless, this study failed to observe any associations between physical activity and the CSA of paraspinal muscles. Fat infiltration is a sign of muscle atrophy $[9,10]$ that may occur prior to any reduction in a muscle CSA. For instance, replacement of muscle with fat, while changing the function of the muscle, may not significantly alter its CSA [21]. No previous study has examined the association between physical activity levels and fat replacement of paraspinal muscles. Histological studies have demonstrated concordance between intermuscular adipose tissue detected by MRI and intra-operative specimens of paraspinal muscles [22], with other studies corroborating MRI as a valid method of identifying the amount of fat in the skeletal muscles [23,24]. Severe fat infiltration of multifidus has strongly been associated with clinical endpoints, such as ever having had low back pain or low back pain in the last year [9]. Using proton MR spectroscopy, a previous study found that compared to 25 asymptomatic controls, participants with chronic low back pain had a significantly higher fat content in multifidus [10]. The current study has demonstrated that independent of age, gender and BMI, low levels of physical activity were associated high fat content of multifidus. Adjusting for the chronic pain grade did not significantly alter the results of the study. Therefore, in a population not selected on the basis of chronic back pain, physical inactivity may be associated with higher fat content, rather than a change in the CSA of multifidus. A similar direction of results was observed between physical inactivity and a high fat content of erector spinae, although this did not reach statistical significance. This may reflect the modest sample size and larger cohorts may help to confirm or refute this relationship.

Finally, this study demonstrated that lower levels of physical activity were associated with an increased risk for high-intensity low back pain and disability in community-based adults. A recent systematic review of 17 studies concluded that the relationship between physical activity and low back pain was too heterogeneous to reach any conclusion [4]. However, on closer scrutiny of this systematic review [4], all adult studies reported significant associations between low physical activity and an increased risk of low back pain [25-27], while inconsistent relationships were more common in studies examining school children [28-33]. Our results provide further support that in an adult population, physical inactivity is associated with high-intensity low back pain and disability.

This study was limited by its modest sample size and predominance of female participants (68.1\%), so larger studies incorporating more male participants are required to substantiate the generalisability of our findings. Moreover, this study cannot determine causality. That is, whether low levels of physical activity are the cause or result of a narrow disc, high multifidus fat content or low back pain/disability cannot be determined from 
these data. Longitudinal studies will help to clarify such issues. However, it is more biologically plausible that a narrow intervertebral disc, high fat content of multifidus or low back pain/disability were the result of physical inactivity, rather than vice versa. Indeed, physical activity was measured between 2005 and 2008, which preceded the MRI spinal structure and low back pain assessment that was performed between 2011 and 2012. It has been shown that this intensity of exercise captured by questionnaire tends to better approximate habitual physical activity levels [14]. Based on validated methods, we employed a semi-quantitative method for assessing fat replacement of paraspinal muscles $[9,15,34]$ and assessed not only low back pain, but a composite score of pain and disability $[16,17]$. Although we acknowledge the potential for these methods to result in misclassification, this non-differential misclassification would have reduced this study's ability to show any significant associations. Additionally, the Chronic Pain Grade Questionnaire provides a composite score of pain and disability, but does not characterise pain (for example radicular and/or mechanical pain). Moreover, our means of assessing activity levels were derived from asking subjects the number of days in the fortnight they had participated in strenuous activities that had resulted in them sweating or experiencing shortness of breath for at least 20 minutes duration. A quantitative measure of physical activity such as measurement via an accelerometer may help to substantiate the findings in this study. This study had MRI scans performed sporadically throughout the day in an attempt to mitigate any diurnal variation that may influence intervertebral disc height. Any diurnal pattern of intervertebral disc height variability would have once again introduced non-differential misclassification into the study, reducing the chances of finding statistically significant results. While we have selected a community-based population, this study had a selection bias towards overweight and obese adults. This may limit the generalisability of our findings to those of normal weight. Finally, we assessed muscle CSA and fat replacement at the level of L3/4 disc level on the left side. This anatomical region was chosen as it approximated a mid-point of the lumbar spine. Analyses at each individual level would have introduced the issue of multiple testing and was therefore avoided. Nevertheless, it is possible that physical activity may have differential effects on paraspinal muscle properties at different spinal levels. The left side was arbitrarily chosen as we presumed that it may have greater fat content, being the non-dominant side in most individuals. Nevertheless, the correlation between muscle CSA between the left and right sides for multifidus and erector spinae was strong $(r=0.87$ and 0.92 , respectively). Similarly, the correlation between the left and right sides for multifidus and erector spinae fat infiltration was also strong
( $\mathrm{r}=0.82$ and 0.95 , respectively). We performed post hoc analyses and demonstrated similar associations between physical activity and right-sided paraspinal muscle properties (data not shown).

\section{Conclusions}

This study of community-based adults demonstrated a dose-response relationship between physical inactivity and a narrow intervertebral disc as well as high multifidus fat content. We also found that physical inactivity was associated with high-intensity pain and disability. Longitudinal studies are required to determine cause and effect between these associations.

\section{Abbreviations}

BMI: body mass index; Cl: confidence interval; CSA: cross-sectional area; ICC: intra-class correlation coefficient; MRI: magnetic resonance imaging; OR: odds ratio; SEM: standard error of the mean.

\section{Competing interests}

The authors declare that they have no competing interests.

\section{Authors' contributions}

AJT contributed to study design, data analyses, and manuscript preparation. DMU contributed to funding, initial recruitment, study design, data acquisition, and manuscript preparation. YW contributed to data acquisition, data analyses, and manuscript preparation. AEW contributed to funding, initial recruitment, study design, data analyses, and manuscript preparation. RO'S contributed to data acquisition and manuscript preparation. GJ contributed to funding, initial recruitment, data acquisition, and manuscript preparation. FMC contributed to funding, initial recruitment, study design, data acquisition, data analyses, and manuscript preparation. All authors read and approved the manuscript.

\section{Authors' information}

Andrew J Teichtahl and Donna M Urquhart are joint first authors.

\section{Acknowledgements}

A.J.T. is the recipient of the NHMRC Early Career Fellowship (\#1073284). D.U., Y.W. and A.E.W. are the recipients of NHMRC Career Development Fellowships (Clinical level 1 \#1011975, Clinical level 1 \#1065464 and Clinical Level 2 \#1063574, respectively). The work was funded by the Monash University Strategic Grant Scheme (PAG001).

\section{Author details}

${ }^{1}$ Department of Epidemiology and Preventive Medicine, School of Public Health and Preventive Medicine, Monash University, Alfred Hospital, 99 Commercial Road, Melbourne, VIC 3004, Australia. ${ }^{2}$ Baker IDI Heart and Diabetes Institute, 75 Commercial Road, Melbourne, VIC 3004, Australia. ${ }^{3} \mathrm{MRI}$ Department, Healthcare Imaging Services, Epworth Hospital, 89 Bridge Road, Richmond, VIC 3121, Australia. ${ }^{4}$ Department of Medicine, Central Clinical School, Monash University, 89 Commercial Road, Melbourne, VIC 3004, Australia. ${ }^{5}$ Menzies Research Institute, 17 Liverpool Street, Hobart, TAS 7000, Australia.

Received: 19 October 2014 Accepted: 17 April 2015

Published online: 07 May 2015

\section{References}

1. Hoy D, March L, Brooks P, Blyth F, Woolf A, Bain C, et al. The global burden of low back pain: estimates from the Global Burden of Disease 2010 study. Ann Rheum Dis. 2014;73:968-74. doi:10.1136/annrheumdis-2013-204428. Epub 2014 Mar 24

2. Holth HS, Werpen HK, Zwart JA, Hagen K. Physical inactivity is associated with chronic musculoskeletal complaints 11 years later: results from the Nord-Trondelag Health Study. BMC Musculoskelet Disord. 2008;9:159. doi:10.1186/471-2474-9-159. 
3. Chen SM, Liu MF, Cook J, Bass S, Lo SK. Sedentary lifestyle as a risk factor for low back pain: a systematic review. Int Arch Occup Environ Health. 2009:82:797-806. doi:10.1007/s00420-009-0410-0. Epub 2009 Mar 20.

4. Sitthipornvorakul E, Janwantanakul P, Purepong N, Pensri P, van der Beek AJ. The association between physical activity and neck and low back pain: a systematic review. Eur Spine J. 2011;20:677-89. doi:10.1007/s00586-010-1630-4. Epub 2010 Nov 27.

5. Sjolie AN. Persistence and change in nonspecific low back pain among adolescents: a 3-year prospective study. Spine (Phila Pa 1976). 2004;29:2452-7.

6. Holguin N, Uzer G, Chiang FP, Rubin C, Judex S. Brief daily exposure to low-intensity vibration mitigates the degradation of the intervertebral disc in a frequency-specific manner. J Appl Physiol (1985). 2011;111:1846-53. doi:10.152/japplphysiol.00846.2011. Epub 2011 Sep 29.

7. Wuertz K, Godburn K, MacLean JJ, Barbir A, Donnelly JS, Roughley PJ, et al. In vivo remodeling of intervertebral discs in response to short- and long-term dynamic compression. J Orthop Res. 2009;27:1235-42. doi:10.002/jor.20867.

8. Castro MJ, Apple Jr DF, Hillegass EA, Dudley GA. Influence of complete spinal cord injury on skeletal muscle cross-sectional area within the first 6 months of injury. Eur J Appl Physiol Occup Physiol. 1999;80:373-8.

9. Kjaer P, Bendix T, Sorensen JS, Korsholm L, Leboeuf-Yde C. Are MRI-defined fat infiltrations in the multifidus muscles associated with low back pain? BMC Med. 2007:5:2.

10. Mengiardi B, Schmid MR, Boos N, Pfirrmann CW, Brunner F, Elfering A, et al. Fat content of lumbar paraspinal muscles in patients with chronic low back pain and in asymptomatic volunteers: quantification with MR spectroscopy. Radiology. 2006;240:786-92.

11. Urquhart DM, Kurniadi I, Triangto K, Wang Y, Wluka AE, O'Sullivan R, et al. Obesity is associated with reduced disc height in the lumbar spine but not at the lumbosacral junction. Spine (Phila Pa 1976). 2014;39:E962-6.

12. Giles GG, English DR. The Melbourne collaborative cohort study. IARC Sci Publ. 2002;156:69-70.

13. Hanna F, Teichtahl AJ, Bell RJ, Davis SR, Wluka AE, O'Sullivan R, et al. The cross-sectional relationship between fortnightly exercise and knee cartilage properties in healthy adult women in midlife. Menopause. 2007;14:830-4.

14. Richardson MT, Ainsworth BE, Jacobs DR, Leon AS. Validation of the Stanford 7-day recall to assess habitual physical activity. Ann Epidemiol. 2001;11:145-53.

15. Kader DF, Wardlaw D, Smith FW. Correlation between the MRI changes in the lumbar multifidus muscles and leg pain. Clin Radiol. 2000;55:145-9.

16. Von Korff M, Ormel J, Keefe FJ, Dworkin SF. Grading the severity of chronic pain. Pain. 1992;50:133-49.

17. Smith BH, Penny Kl, Purves AM, Munro C, Wilson B, Grimshaw J, et al. The chronic pain grade questionnaire: validation and reliability in postal research. Pain. 1997;71:141-7.

18. Livshits G, Popham M, Malkin I, Sambrook PN, Macgregor AJ, Spector T, et al. Lumbar disc degeneration and genetic factors are the main risk factors for low back pain in women: the UK Twin Spine Study. Ann Rheum Dis. 2011;70:1740-5. doi:10.136/ard.2010.137836. Epub 2011 Jun 6

19. Kettler A, Wilke HJ. Review of existing grading systems for cervical or lumbar disc and facet joint degeneration. Eur Spine J. 2006;15:705-18. Epub 2005 Sep 20

20. Pfirrmann CW, Metzdorf A, Zanetti M, Hodler J, Boos N. Magnetic resonance classification of lumbar intervertebral disc degeneration. Spine (Phila Pa 1976). 2001;26:1873-8.

21. D'Hooge R, Cagnie B, Crombez G, Vanderstraeten G, Dolphens M, Danneels L. Increased intramuscular fatty infiltration without differences in lumbar muscle cross-sectional area during remission of unilateral recurrent low back pain. Man Ther. 2012;17:584-8. doi:10.1016/j.math.2012.06.007. Epub 2012 Jul 10.

22. Rossi A, Zoico E, Goodpaster BH, Sepe A, Di Francesco V, Fantin F, et al. Quantification of intermuscular adipose tissue in the erector spinae muscle by MRI: agreement with histological evaluation. Obesity (Silver Spring). 2010;18:2379-84. doi:10.1038/oby.2010.48. Epub 2010 Mar 18.

23. Mitsiopoulos N, Baumgartner RN, Heymsfield SB, Lyons W, Gallagher D, Ross R. Cadaver validation of skeletal muscle measurement by magnetic resonance imaging and computerized tomography. J Appl Physiol (1985). 1998;85:115-22.

24. Phoenix J, Betal D, Roberts N, Helliwell TR, Edwards RH. Objective quantification of muscle and fat in human dystrophic muscle by magnetic resonance image analysis. Muscle Nerve. 1996;19:302-10.
25. Brown WJ, Mishra G, Lee C, Bauman A. Leisure time physical activity in Australian women: relationship with well being and symptoms. Res Q Exerc Sport. 2000;71:206-16.

26. Bjorck-van Dijken C, Fjellman-Wiklund A, Hildingsson C. Low back pain, lifestyle factors and physical activity: a population based-study. J Rehabil Med. 2008:40:864-9. doi:10.2340/16501977-0273.

27. Heneweer H, Vanhees L, Picavet HS. Physical activity and low back pain: a U-shaped relation? Pain. 2009;143:21-5. doi:10.1016/j.pain.2008.12.033. Epub 2009 Feb 12

28. Wedderkopp N, Kjaer P, Hestbaek L, Korsholm L, Leboeuf-Yde C. High-level physical activity in childhood seems to protect against low back pain in early adolescence. Spine J. 2009;9:134-41. doi:10.1016/j.spinee.2008.02.003. Epub 2008 May 20.

29. Auvinen J, Tammelin T, Taimela S, Zitting P, Karppinen J. Associations of physical activity and inactivity with low back pain in adolescents. Scand J Med Sci Sports. 2008;18:188-94. Epub 2007 May 9.

30. Diepenmaat AC, van der Wal MF, de Vet HC, Hirasing RA. Neck/shoulder, low back, and arm pain in relation to computer use, physical activity, stress, and depression among Dutch adolescents. Pediatrics. 2006;117:412-6.

31. Kujala UM, Taimela S, Viljanen T. Leisure physical activity and various pain symptoms among adolescents. Br J Sports Med. 1999:33:325-8.

32. Sjolie AN. Associations between activities and low back pain in adolescents. Scand J Med Sci Sports. 2004;14:352-9.

33. Mikkelsson LO, Nupponen H, Kaprio J, Kautiainen H, Mikkelsson M, Kujala UM. Adolescent flexibility, endurance strength, and physical activity as predictors of adult tension neck, low back pain, and knee injury: a 25 year follow up study. Br J Sports Med. 2006;40:107-13.

34. Parkkola R, Rytokoski U, Kormano M. Magnetic resonance imaging of the discs and trunk muscles in patients with chronic low back pain and healthy control subjects. Spine (Phila Pa 1976). 1993;18:830-6.

\section{Submit your next manuscript to BioMed Central and take full advantage of:}

- Convenient online submission

- Thorough peer review

- No space constraints or color figure charges

- Immediate publication on acceptance

- Inclusion in PubMed, CAS, Scopus and Google Scholar

- Research which is freely available for redistribution 